Background Undifferentiated arthritis could be an early stage or forme fruste of a definite rheumatic disease.

Objectives The purpose of this poster is to describe the evolution of 40 patients ( $>16$ years) in our hospital, with seronegative mono-oligoarthritis, HLA B27 negative, without axial involvement, that at the onset of the disease did not meet the criteria to be included in any other defined arthropathy.

Methods Forty patients (21 females and 19 males) with seronegative undifferentiated oligoarthritis, without axial involvement, that could not be typified as rheumatic disease of the rheumatoid, psoriatic, enteropathic, gouty, or reactive type, were studied retrospectively. In all patients the presence of crystals in their synovial fluid was discared as well as skin or eye symptoms, infectious diseases (Salmonella, Shigella, Yersinia, Ureaplasma and mycobacteria) or associated connective-tissue disease.

Results The mean age at the onset of oligoarthritis was 42.1 years (44.9 for males and 36.3 for females). The average duration of follow-up was 11.1 years (range 24-1). Eight (28\%) of the 40 patients had just one joint affected while the remaining patients presented with 4 or less involved joints. In 8 of our cases $(20 \%)$ the disease resolved spontaneously. In 12 patients (30\%), a definitive diagnosis was made after an average of 4.1 years (range 13-1) from the onset to the final diagnosis. Among these 12 patients, 5 were diagnosed as gout, 2 as arthropathy associated with inflammatory bowel disease, 2 as psoriatic arthritis, 2 as rheumatoid arthritis and one case of SAPHO associated arthropathy. In the remaining patients the disease followed a chronic course and no improvement was detected. Only 16 of the 40 patients received specific antirheumatic drug therapy: 10 of the 20 cases in which the disease took a chronic course and for which no diagnosis was reached.

Conclusion We would like to stress that no rheumatologist would deny the difficulty implied in diagnosing and managing patients with undifferentiated mono-oligoarthritis that do not fit into any typified rheumatic disease. Nevertheless, although 50\% of our patients have not yet been definitively diagnosed after a 6.5 year average follow-up, a final diagnosis has been reached for $30 \%$ of the total number in a 4.1 -year period on the average.

\section{SAT0014 TREATMENT OF PSORIATIC ARTHRITIS AND PSORIASIS VULGARIS WITH THE TUMOUR NECROSIS FACTOR BLOCKER INFIXIMAB}

EE Cauza, MS Spak, KC Cauza, UH Hanusch-Enserer, AD Dunky, EW Wagner. Department of Rheumatology/Medicine 5, Wilhelminenspital, Vienna, Austria

\subsection{6/annrheumdis-2001.366}

Background Tumour necrosis factor a (TNF a), a proinflammatory cytokine, is considered to be one of the important mediators in the pathogenesis of psoriasis and psoriatic arthritis (PsA) and may therefore be a useful target for specific immunotherapy. Treatment of rheumatoid arthritis with TNF inhibitors has proven to be extremely effective, so the aim of our study was to evaluate the efficacy and safety of multiple infusions with a humanised anti-tumour necrosis factor a monoclonal antibody (infliximab) in PsA and Psoriasis.

Objectives

Methods Over a period of 22 weeks, nine consecutive patients (5 men, 4 women, mean age 57.3 years, mean duration of PsA/ Psoriasis 17.2/18.1 years) who had both active psoriasis and PsA and fulfilled diagnostic criteria were included in this study.
Treatment resistant patients with active disease received infusions of $3 \mathrm{mg} / \mathrm{kg}$ infliximab (at week 0, 2, 614 , and 22). The primary endpoints included the changes from baseline to week 22 in the swollen and tender joint count and the improvements of the psoriasis activity (Pasi).

Results The treatment was well tolerated, only one mild side effect was noted. Significant improvements were observed after 2 weeks in the decrease of swollen and tender joint count, which fell from a median of 5.3 and 17.88 at study entry to a median of 1.44 and 9.77 by week $2(\mathrm{p}=0.02 / \mathrm{p}=0.002)$ and maintained up to week 22. Over the treatment period of twenty two weeks the mean Pasi changed statistically significant from 19.04 to 4.39 ( $\mathrm{p}=0.002)$. The improvements of the main parameters are summarised in Table 1.

Abstract SAT0014 Table 1 Values (mean) of the main parameters in our 9 patients, before treatment (T0), after 2 weeks (T2), after 14 weeks (T14) and after 22 weeks (T22), statistical significance $\left(\mathrm{p}^{*}\right)$ determined by student t-test of the change of baseline $(p<0.05)$

\begin{tabular}{llllll}
\hline & T0 & T2 & T14 & T22 & p \\
\hline Pasi & 19.04 & 7.41 & 1.66 & 4.39 & $\mathrm{p}=0.0021$ \\
MGST & 52.77 & 23.33 & 23.33 & 24.0 & $\mathrm{p}=0.0114$ \\
SJC & 5.33 & 1.44 & 2.77 & 2.8 & $\mathrm{p}=0.046$ \\
TJC & 17.88 & 9.77 & 9.44 & 8.4 & $\mathrm{p}=0.00321$ \\
CRP & 16.04 & 7.8 & 4.02 & 4.32 & $\mathrm{p}=0.041$ \\
ESR & 34.44 & 14.66 & 18.0 & 16.2 & $\mathrm{p}=0.015$ \\
VAS & 5.88 & 3.33 & 3.77 & 3.6 & $\mathrm{p}=$ n.s. \\
\hline
\end{tabular}

Pasi (Psoriasis Area and the Severity Index), MGST (Morning stiffness in minutes), SJC (Swollen joint count), TJC (Tender joint count), CRP (C-reactive protein), ESR (Erythrocyte sedimentation rate) VAS (Assessment of pain).

Conclusion Multiple infusions of infliximab were effective and well tolerated in patients with active psoriasis and PsA. The results provide a good evidence that specific cytokine blockade can be effective in inflammatory disease and offers patients with PsA and psoriasis a new direction of treatment.

\section{SAT0015 UVEITIS AS THE INITIAL CLINICAL MANIFESTATION IN PATIENS WITH SPONDYLOARTHOPATHIES}

${ }^{1} \mathrm{~J}$ Fernández-Melón, ${ }^{1} \mathrm{~S}$ Muñoz-Fernández, ${ }^{2} \mathrm{~V}$ Hidalgo, ${ }^{1} \mathrm{G}$ Bonilla, ${ }^{2} \mathrm{~A}$ Schlincker, ${ }^{2}$ A Fonseca, ${ }^{2} \mathrm{~J}$ Vieitez, ${ }^{1} \mathrm{E}$ Martin-Mola. ${ }^{1}$ Rheumatology Service; ${ }^{2}$ Ophthalmology Service, Hospital Universitario La Paz, Madrid, Spain

10.1136/annrheumdis-2001.367

Background Uveitis can be the first manifestation in patients with spondyloarthropathy (spA).

Objectives To investigate the frecuency and pattern of presentation uveitis as the first clinical manifestation in patients with spA.

Methods In this study we include the patients with uveitis attended simoultaneosly by ophthalmologists and rheumatologists in our Uveitis Clinic between June 1997 and October 2000. A clinical preestablished protocol based on the pattern of uveitis and the patient's symptoms was used to achieve diagnosis in each case. Diagnostic evaluation included, a clinical history, an ophthalmologic examination, hemogram, biochemistry, ESR, a fluorescent treponemal antibody absortion test, urinary test and a chest $\mathrm{x}$-ray. In addition, in patients with previous flares of 